\title{
Neolignanas e análise do óleo essencial das folhas de Piper regnellii (Miq.) C. DC. var. pallescens (C. DC.) Yunck
}

\author{
G.L. Pessini', B.P. Dias Filho², C.V. Nakamura ${ }^{2}$, A.G. Ferreira ${ }^{3}$, D.A.G. Cortez ${ }^{1 *}$ \\ ${ }^{1}$ Departamento de Farmácia e Farmacologia, Universidade Estadual de Maringá, 87020-900, Maringá, PR, Brasil, \\ ${ }^{2}$ Departamento de Análises Clínicas, Universidade Estadual de Maringá, 87020-900, \\ Maringá, PR, Brasil, \\ ${ }^{3}$ Departamento de Química, Universidade Federal de São Carlos, Caixa Postal 676, \\ 13565-955, São Carlos, SP, Brasil
}

\begin{abstract}
RESUMO: O óleo essencial das folhas de Piper regnellii (Miq.) C. DC. var. pallescens (C. DC.) Yunck Piparaceae, coletadas no Horto de Plantas Medicinais da Universidade Estadual de Maringá, foi obtido por hidrodestilação. Uma análise preliminar por CG/EM e RMN ${ }^{13} \mathrm{C}$ foi realizada. O $\beta$ mirceno (70\%) foi identificado como componente majoritário através da comparação dos espectros de massa e RMN ${ }^{13} \mathrm{C}$ com dados da literatura. Quatro neolignanas foram isoladas do extrato hidroetanólico das folhas e identificadas: eupomatenóide-6, eupomatenóide-5, eupomatenóide-3 e conocarpano. As estruturas dessas substâncias foram estabelecidas por meio de estudos de $\mathrm{RMN}{ }^{1} \mathrm{H}$ e ${ }^{13} \mathrm{C},{ }^{1} \mathrm{H}$ x ${ }^{1} \mathrm{H}$ - COSY, HETCOR, HMBC, gNOE e EM.
\end{abstract}

Unitermos: Piper regnellii, Piperaceae, neolignanas, óleo essencial, CG/EM.

\begin{abstract}
Neolignans and the analysis of the essential oil of Piper regnellii (Miq.) C. DC. var. pallescens (C. DC.) Yunck leaves". The essential oil of Piper regnellii (Miq.) C. DC. var. pallescens (C. DC.) Yunck Piparaceae leaves, which were collected at a tree farm named Horto de Plantas Medicinais of the Universidade Estadual de Maringá, was obtained by hydrodistillation. A preliminary analysis by GC/MS was carried out. $\beta$-mirceno (70\%) was identified as the main constituent by comparing MS and ${ }^{13} \mathrm{C}$ NMR with the literature data. Four neolignans were isolated from the leaves and identified: eupomatenoid-6, eupomatenoid-5, eupomatenoid-3 and conocarpan. Their structures were established by extensive ${ }^{1} \mathrm{H}$ and ${ }^{13} \mathrm{C}$ NMR, ${ }^{1} \mathrm{H} \mathrm{x}{ }^{1} \mathrm{H}-\mathrm{COSY}$, HETCOR, HMBC, gNOE and MS spectral studies.
\end{abstract}

Keywords: Piper regnellii, Piperaceae, neolignans, essential oil, GC/MS

\section{INTRODUCÃO}

Substâncias isoladas de diferentes espécies de Piper têm demonstrado interesse nos últimos anos, devido às atividades biológicas. Diversas neolignanas isoladas de espécies de Piperaceae apresentaram atividades biológicas como anti-PAF, antifúngica e inseticida (Sartorelli et al., 2001).

Piper regnellii (Miq) C. DC. var. pallescens (C. DC.) Yunck (Piperaceae) é uma espécie herbácea conhecida popularmente como pariparoba e está distribuída nas regiões tropicais e subtropicais do mundo (Cronquist, 1981). As folhas e raízes são usadas na forma de extratos, infusões ou emplastros para o tratamento de feridas, redução de inchaços e irritações na pele (Corrêa, 1984). Screening realizado com plantas medicinais reportou atividade antimicrobiana do extrato hidroetanólico das folhas $P$. regnellii (Miq) C. DC sobre as bactérias Staphylococcus aureus e Bacillus subtilis, fungos Candida krusei e C. tropicalis (Holetz et al., 2002).

Em estudo prévio, Benevides et al. (1999) isolaram diferentes neolignanas presentes no extrato em acetato de etila das raízes de $P$. regnellii (Miq.) C. DC., dentre as quais o conocarpano, eupomatenóide-3, eupomatenóide-5 e eupomatenóide-6.

Estudos posteriores sobre constituintes voláteis relataram a atividade antimicrobiana do óleo essencial das folhas de P. regnellii (Miq.) C. DC. sobre o Staphylococcus aureus e Candida albicans. Os constituintes químicos do óleo essencial foram identificados e sua concentração relativa estabelecida por análise espectral de CG-EM. A substância majoritária do óleo de $P$. regnellii foi o mirceno (52,6 \%), seguido pelo linalol (15,9\%), $\beta$-cariofileno (8,5 $\%),(E)$-nerolidol (4,2 \%) e limoneno (4,1 \%) (Constantin et al., 2001).

Neolignanas isoladas das folhas de Piper regnellii (Miq) C. DC. var. pallescens (C. DC.) Yunck, 
como eupamotenóides-6 e eupomatenóide-5, mostraram boa atividade sobre $S$. aureus com CIM de $1.56 \mu \mathrm{g} / \mathrm{mL}$ e $3.12 \mu \mathrm{g} / \mathrm{mL}$, respectivamente. Ambas substâncias apresentaram atividade em $B$. subtilis com CIM de 3.12 $\mu \mathrm{g} / \mathrm{mL}$. O conocarpano foi ativo sobre $S$. aureus e $B$. subtilis com CIM de $6.25 \mu \mathrm{g} / \mathrm{mL}$ (Pessini et al., 2003).

Devido ao uso popular, o estudo da composição química das folhas de Piper regnellii (Miq) C. DC. var. pallescens (C. DC.) Yunck é necessário, contribuindo para futura padronização de fitoterápicos originários desta espécie vegetal.

No presente trabalho, é apresentado o estudo fitoquímico, objetivando o isolamento e identificação de neolignanas e a realização de uma análise preliminar da composição química do óleo essencial, por CG/EM e RMN ${ }^{13} \mathrm{C}$, obtido das folhas da $P$. regnellii (Miq) C. DC. var. pallescens (C. DC.) Yunck.
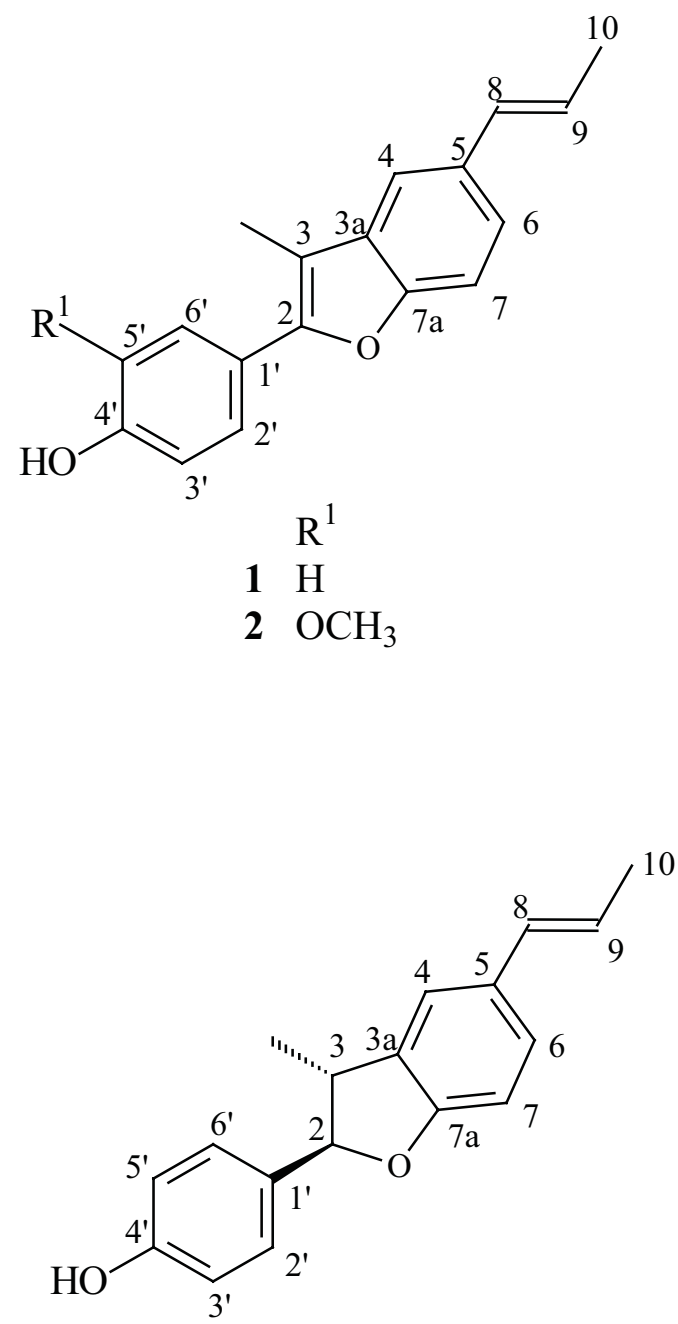

4

\section{MATERIAL E MÉTODOS}

\section{Procedimentos experimentais gerais}

Os espectros no infravermelho foram registrados em espectrômetro BOMEN - MV 100, Hartmann \& Braun- Michelson. Os espectros de RMN foram registrados a $300 \mathrm{MHz}$ para ${ }^{1} \mathrm{H}$ e $75,45 \mathrm{MHz}$ para ${ }^{13} \mathrm{C}$ em espectrômetro VARIAN modelo MERCURY PLUS. Foi utilizado $\mathrm{CDCl}_{3}$ como solvente e TMS como referência interna. Para a obtenção de espectro no ultravioleta foi utilizado o aparelho VARIAN C ary 1E. Os espectros de massa por electrospray foram obtidos em espectrômetro Micromass Quattro LC e por impacto eletrônico em um equipamento Shimadzu modelo QP2000A.

As cromatografias em coluna foram realizadas em colunas de vidro, utilizando-se sílica gel 60 (70-230 mesh ASTM) da Merck e na cromatografia no modo

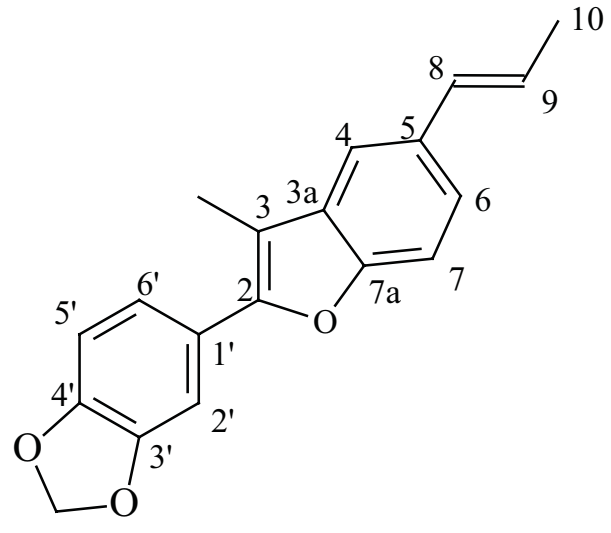

3<smiles>[2H]C(I)=C([2H])CC(=C)C=[18O]</smiles>

5

Figura 1. Estruturas das substâncias analisadas nas folhas de Piper regnelli: eupomatenóide-6 (1), eupomatenóide-5 (2), eupomatenóide-3 (3), conocarpano (4) e $\beta$-mirceno (5). 
flash sílica gel 60 (230-400 mesh ASTM) da Merck. Nas cromatografias em placa utilizou-se sílica gel 60 $\mathrm{GF}_{254}$. As revelações deste tipo de cromatografia foram efetuadas por irradiação no ultravioleta $(\lambda=254$ e 366 $\mathrm{nm})$ e por pulverizações com solução de $\mathrm{H}_{2} \mathrm{SO}_{4} / \mathrm{MeOH}$ $1: 1$, seguidas de aquecimento a $105^{\circ} \mathrm{C}$ até a revelação das substâncias químicas.

\section{Material vegetal}

As folhas de Piper regnellii (Miq.) C. CD. var. pallescens (C. DC.) Yunck foram coletadas em agosto de 2001 no Horto de Plantas Medicinais Prof ${ }^{a}$. Irenice Silva do Campus da Universidade Estadual de Maringá. O material vegetal foi identificado por Marília Borgo, do Departamento de Botânica da Universidade Federal do Paraná e uma exsicata deste material (nº. HUM 8392) foi depositada no Herbário da Universidade Estadual de Maringá, Paraná, Brasil.

\section{Obtenção do óleo essencial}

A extração do óleo essencial foi realizada por arraste a vapor d'água, como descrito pela Farmacopéia Brasileira (1988). Foram utilizadas as folhas frescas de $P$. regnellii var. pallescens. A densidade relativa do óleo essencial foi determinada pela técnica descrita na Farmacopéia Brasileira (1988).

\section{Análise do óleo essencial.}

O óleo essencial obtido por arraste a vapor de folhas frescas de Piper regnellii foi analisado por CG/ EM em um equipamento Shimadzu modelo QP2000A. A coluna cromatográfica utilizada foi do tipo capilar de sílica fundida com fase estacionária SE-30 de $25 \mathrm{~m}$ de comprimento e 0,20 mm de diâmetro interno, sendo utilizado Hélio como gás carreador. As temperaturas foram de $220^{\circ} \mathrm{C}$ no injetor e $300^{\circ} \mathrm{C}$ no detector. A temperatura do forno foi programada de 100 a $300^{\circ} \mathrm{C}$, com acréscimo de $10^{\circ} \mathrm{C}$ a cada minuto.

A identificação da substância majoritária presente no óleo essencial foi feita por meio da análise de espectro de massas, espectros de ${ }^{1} \mathrm{H}$ e ${ }^{13} \mathrm{C}$ e por comparação com os dados da literatura.

\section{Isolamento dos constituintes}

As folhas foram coletadas, secas em estufa de ar circulante a $45^{\circ} \mathrm{C}$ e moída em moinho de facas Tecnal Marconi ${ }^{\circledR}$ (modelo TE 048), utilizando-se uma peneira de $1 \mathrm{~mm}$ de diâmetro. O pó das folhas secas (200 g) de $P$. regnellii foi extraído com etanol:água $(9: 1) \mathrm{v} / \mathrm{v}$ a temperatura ambiente até esgotamento total dos princípios ativos. O solvente foi removido em evaporador rotativo em temperatura máxima de $40^{\circ} \mathrm{C}$ e após liofilização, obteve-se um resíduo, o qual foi solubilizado em acetato de etila. Este solvente foi evaporado à pressão reduzida e originou o extrato em acetato de etila $(15,23 \mathrm{~g})$, que foi analisado por meio de CCD e RMN ${ }^{1} \mathrm{H}$.

Este extrato (15,23 g) foi submetido a cromatografia a vácuo (sílica gel 150 g) e eluído com hexano $(1000 \mathrm{~mL})$, clorofórmio $(1400 \mathrm{~mL})$, clorofórmio/ acetato de etila 19:1 v/v (1000 mL), clorofórmio/acetato de etila 9:1 v/v (700 mL), clorofórmio/acetato de etila 1:1 v/v (500 mL), acetato de etila $(500 \mathrm{~mL})$, acetona (700 $\mathrm{mL})$, metanol (1400 mL) e metanol/água 9:1 v/v (1800 $\mathrm{mL})$ e as frações resultantes foram analisadas por CCD e RMN ${ }^{1} \mathrm{H}$. A fração hexânica (2.4 g) (F1) foi fracionada por coluna cromatográfica em sílica gel 60 (70-230 mesh), eluída com hexano, hexano/clorofórmio 49:1, 19:1, 9:1 e 1:1 v/v, clorofórmio, acetato de etila, acetona e metanol. Obteve-se 72 frações, dentre as quais as de número FH 20, FH 15 e FH 10 resultaram na obtenção das substâncias 1 (98,5 mg), 2 (82,9 mg) e 3 (55,1 mg) respectivamente. As frações $\mathrm{FH}$ 40-44 reunidas (328 mg) e cromatografadas em sílica gel 60 (230-400 mesh), utilizando-se como fase móvel hexano:diclorometano: acetato de etila 12:7:1 v/v/v, forneceram a substância 4 (181,3 mg). 1, 2, 3 e 4 foram identificadas como eupomatenóide-6, eupomatenóide-5, eupomatenóide-3 e conocarpano (Figura 1) respectivamente após análise de dados fornecidos por métodos espectroscópicos (UV, EIEM, RMN ${ }^{1} \mathrm{H}, \mathrm{RMN}{ }^{13} \mathrm{C}, \mathrm{H}-\mathrm{HCOSY}$, gNOE, HETCOR e HMBC) e por comparação com dados da literatura (Chauret et al., 1996; Achenbach et al., 1987; Snider et al., 1997; Maxwell et al., 1999).

\section{Eupomatenóide-6 (1)}

Aspecto físico: cristal branco. P.F. $150-151^{\circ} \mathrm{C}$ (lit.: $148-151^{\circ} \mathrm{C}$ ) (Achenbach et al., 1987). UV $\lambda_{\text {max }}$ $\mathrm{CHCl}_{3}$, nm (log $\left.\varepsilon\right): 234$ (9,46); 258 (9,45); 300 (9;43). IV $v_{\max } \mathrm{cm}^{-1}: 3277,2957,2928,2853,1609,1581,1511$, 1440, 1237. RMN ${ }^{1} \mathrm{H}\left(300 \mathrm{MHz}, \mathrm{CDCl}_{3}\right): \delta 7,68(\mathrm{~d}, J=$ 8,7 Hz, H-2' e H-6'); 7,43 (d, $J=1,4$ Hz, H-4); 7,36 (d, $J=8,7 \mathrm{~Hz}, \mathrm{H}-7$ ); 7,27 (dd, $J=8,7$ e 1,5 Hz, H-6); 5,06 (s, OH-4'); 6,94 (d, $J=8,6$ Hz, H-3' e H-5’); 6,52 (dd, $J$ = 15,6 e 1,5 Hz, H-8); 6, 25 (dq, $J=15,6$ e 6,6 Hz, H-9); $2,42\left(\mathrm{~s}, \mathrm{CH}_{3}-3\right)$ e 1,90 (dd, $J=6,6$ e $1,5 \mathrm{~Hz}, \mathrm{CH}_{3}-10$ ). RMN ${ }^{13} \mathrm{C}\left(75,5 \mathrm{MHz}, \mathrm{CDCl}_{3}\right)$ : Tabela 1. EM m/z (int. rel.\%): [M] 264 (100), 263 (20), 237 (7), 221 (7), 132 (7), 121 (9), 115 (5), 39 (5).

\section{Eupomatenóide-5 (2)}

Aspecto físico: cristal branco. P.F. $113-115^{\circ} \mathrm{C}$ (lit.: $\left.114-115^{\circ} \mathrm{C}\right)\left(\right.$ Chauret et al., 1996). UV $\lambda_{\text {max }} \mathrm{CHCl}_{3}$, nm (log $\varepsilon)$ : 235 (9,60); 258 (9,70); 314 (9,62). IV $v_{\max } \mathrm{cm}^{-}$ ${ }^{1}$ : 3473, 2970, 2925, 2872, 2845, 1580, 1516, 1459, 1430 , 1262. $\mathrm{RMN}^{1} \mathrm{H}\left(300 \mathrm{MHz}, \mathrm{CDCl}_{3}\right): \delta 7,43$ (d, $J=1,8 \mathrm{~Hz}$, H-4); 7,37 (d, $J=8,4$ Hz, H-7); 7,32 (d, $J=1,8$ Hz, H-6'); 7,29 (dd, $J=8,1$ e 1,8 Hz, H-2'); 7,27 (dd, $J=8,4$ e 1,8 Hz, H-6); 7,03 (d, $J=8.1 \mathrm{~Hz}, \mathrm{H}-3$ '); 6,52 (dd, $J=15,9$ 


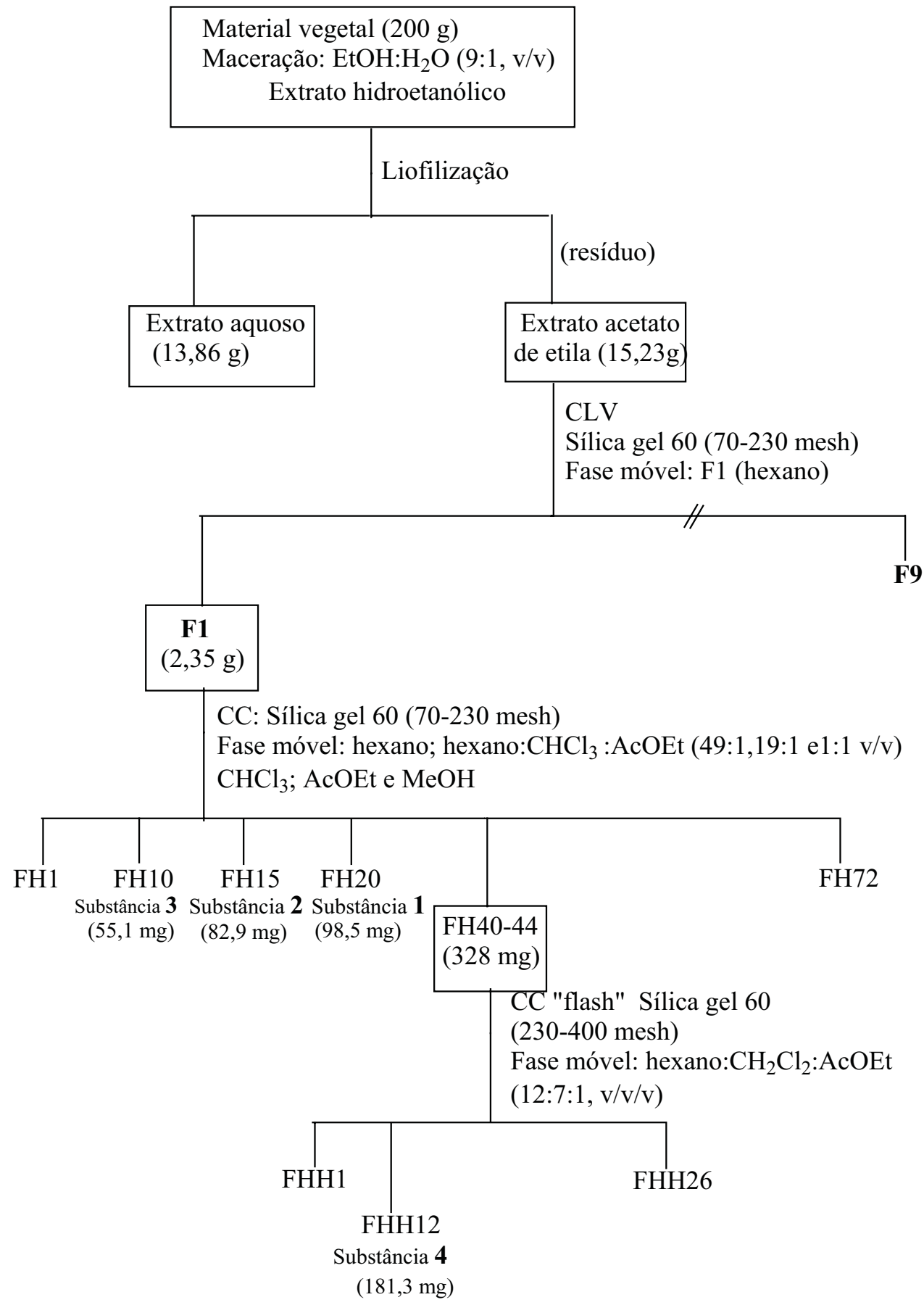

Figura 2. Fluxograma do fracionamento do extrato hidroalcoólico das folhas de Piper regnellii (Miq.) C. DC. var. pallescens (C. DC.) Yunck.

e 1,8 Hz, H-8); 6,23 (dq, $J$ = 15,6 e 6,6 Hz, H-9); 5,75 (s, OH-4'); 3,98 (s, $\left.\mathrm{OCH}_{3}\right) ; 2,42$ (s, $\left.\mathrm{CH}_{3}-3\right)$ e 1,90 (dd, $J$ = 6,6 e $\left.1,8 \mathrm{~Hz}, \mathrm{CH}_{3}-10\right)$. $\mathrm{RMN}{ }^{13} \mathrm{C}\left(75,5 \mathrm{MHz}, \mathrm{CDCl}_{3}\right)$ : $\delta$ Tabela 1. EM m/z (int. rel.\%): [M] 294 (100), 293 (6), 279 (8), 251 (7), 147 (6), 55 (4); ESIEM $m / z$ (int. rel.\%): $293(100)[\mathrm{M}-\mathrm{H}]^{-}$.

\section{Eupomatenóide-3 (3)}

Aspecto físico: cristal branco. P.F. $109-111^{\circ} \mathrm{C}$ (lit.: $110-111^{\circ} \mathrm{C}$ ) (Maxwell et al., 1999). UV $\lambda_{\max } \mathrm{CHCl}_{3}$, nm (log $\varepsilon): 240$ (9,82); 257 (9,77); 324 (9,66). IV $v_{\text {max }}$ $\mathrm{cm}^{-1}: 2785,2850,2921,29511502,1460$ 1238. RMN ${ }^{1} \mathrm{H}$ (300 MHz, $\mathrm{CDCl}_{3}$ ): $\delta$ 7,41 (d, $\left.J=1,5 \mathrm{~Hz}, \mathrm{H}-4\right) ; 7,35$ (d, $J$ = 8,7 Hz, H-7); 7,28 (dd, $J=8,7$ e 1,5 Hz, H-6'); 7,26 (d, $J=1,5 \mathrm{~Hz}, \mathrm{H}-2^{\prime}$ ); 7,27 (dd, $J=8,6$ e 1,5 Hz, H-6); 6,90 (d, $J=8,7 \mathrm{~Hz}, \mathrm{H}-5$ ); 6,51 (dd, $J=15,9$ e 1,8 Hz, H-8); 6,21 (dq, $J=15.9$ e 6,6 Hz, H-9); 6,00 (s, $\left.\mathrm{OCH}_{2} \mathrm{O}\right) ; 2,40$ (s, $\mathrm{CH}_{3}-3$ ); e 1,90 (dd, $J=6,6$ e $1,8 \mathrm{~Hz}, \mathrm{CH}_{3}-10$ ). $\mathrm{RMN}$ ${ }^{13} \mathrm{C}\left(75,5 \mathrm{MHz}, \mathrm{CDCl}_{3}\right)$ : $\delta$ Tabela 1. EM $\mathrm{m} / \mathrm{z}$ (int. rel.\%): 
Tabela 1. Dados de $\mathrm{RMN}^{13} \mathrm{C}$ das substâncias $\mathbf{1}^{\mathrm{a}}, \mathbf{2}^{\mathrm{a}, \mathrm{b}}, \mathbf{3}^{\mathrm{a}}$ e $\mathbf{4}^{\mathrm{a}}\left[75,5 \mathrm{MHz}, \mathrm{CDCl}_{3}, \delta(\mathrm{ppm})\right]$

\begin{tabular}{lcccc}
\hline & $\mathbf{C}$ & $\mathbf{2}$ & $\mathbf{3}$ & $\mathbf{4}$ \\
\hline $1{ }^{\prime}$ & 124,5 & 123,9 & 125,6 & 132,4 \\
2 & 151,2 & 151,4 & 151,1 & 12,6 \\
2 & 128,4 & 120,5 & 120,9 & 45,2 \\
3 & 109,8 & 109,9 & 110,2 & 133,0 \\
$3^{\mathrm{a}}$ & 131,6 & 131,7 & 131,5 & 115,5 \\
$3^{\prime}$ & 115,6 & 114,6 & 108,6 & 121,0 \\
4 & 116,2 & 116,2 & 116,2 & 155,8 \\
4 & 155,5 & 146,8 & 147,5 & 131,4 \\
5 & 132,7 & 132,8 & 132,8 & 115,5 \\
5 & 115,6 & 145,8 & 148,1 & 126,4 \\
6 & 122,3 & 122,3 & 122,4 & 109,3 \\
6 & 128,4 & 109,3 & 107,2 & 158,4 \\
7 & 110,7 & 110,7 & 110,7 & 130,8 \\
$7 \mathrm{a}$ & 153,1 & 153,2 & 153,0 & 123,1 \\
8 & 131,4 & 131,4 & 131,4 & 18,3 \\
9 & 124,3 & 124,3 & 124,3 & 17,7 \\
$\mathrm{CH}_{3}-10$ & 18,4 & 18,4 & 18,4 & - \\
$\mathrm{CH}_{3}-3$ & 9,2 & 9,2 & 9,3 & - \\
$\mathrm{CH}_{3} \mathrm{O}$ & - & 56,0 & - & 101,3 \\
$\mathrm{O}_{-}-\mathrm{CH}_{2}-\mathrm{O}$ & - & - & & \\
\hline
\end{tabular}

a Os sinais de átomos de carbono $\mathrm{C}, \mathrm{CH}, \mathrm{CH}_{2}$ e $\mathrm{CH}_{3}$ foram reconhecidos pela análise comparativa dos espectros de RMN ${ }^{13} \mathrm{C}, \mathrm{RMN}{ }^{13} \mathrm{C}-\mathrm{DEPT}$, HETCOR, HMBC, e ${ }^{\mathrm{g}} \mathrm{g}-\mathrm{NOE}$.

$[\mathrm{M}]^{+} 292$ (100), 291 (11), 265 (5), 189 (7), 146 (7), 89, (6), 76 (6).

\section{Conocarpano (4)}

Aspecto físico: cristal branco. P.F. $128-130^{\circ} \mathrm{C}$ (lit.: $128-130{ }^{\circ} \mathrm{C}$ ) (Snider et al., 1997). $[\alpha]^{25}+123^{\circ}$ (c 1.05; MeOH) [lit. [ $\alpha]^{21}+122^{\circ}$ (c 1.03; $\mathrm{MeOH}$ ] (Snider et al., 1997). UV $\lambda_{\text {max }} \mathrm{CHCl}_{3}, \mathrm{~nm}(\log \varepsilon): 235$ (9,57); 267 (9,79); 305 (9,23). IV $v_{\max } \mathrm{cm}^{-1}$ : 3376, 2961, 2930, 2912, 2874, 1615, 1599, 1518, 1490, 1239. RMN ${ }^{1} \mathrm{H}$ : (300 MHz, $\left.\mathrm{CDCl}_{3}\right)$ : $\delta$ 7,30 (d, $J=8,7 \mathrm{~Hz}, \mathrm{H}-2$ 'e H-6'); 7,12 (dl, $J=8,1 \mathrm{~Hz}, \mathrm{H}-6$ ); 7,10 (sl, H-4); 6,83 (d, $J=8.7$ Hz, H-3' e H-5'); 6,76 (d, $J=8.1 \mathrm{~Hz}, \mathrm{H}-7$ ); 6,36 (dd, $J$ $=15,6$ e 1,5 Hz, H-8); 6,11 (dq, $J=15,6$ e 6,6 Hz, H-9); 5,08 (d, $J=9.0$ Hz, H-2); 4,98 (s, OH-4'); 3,39 (m, H3); 1,86 (dd, $J=6,6$ e 1,5 Hz, $\left.\mathrm{CH}_{3}-10\right)$; 1,39 (d, $J=6,9$ $\left.\mathrm{Hz}, \mathrm{CH}_{3}-3\right) . \mathrm{RMN}{ }^{13} \mathrm{C}\left(75,5 \mathrm{MHz}, \mathrm{CDCl}_{3}\right): \delta$ Tabela 1. $\mathrm{EM} \mathrm{m/z} \mathrm{(rel.} \mathrm{int.} \mathrm{\% ):} \mathrm{[M]} 266$ (100), 251 (31), 237 (9), 224 (7), 107 (22), 91 (17), 77 (16), 39 (12).

\section{$\beta$-Mirceno (5)}

Aspecto físico: óleo translúcido. $\mathrm{RMN}{ }^{1} \mathrm{H}$ (300 MHz, $\left.\mathrm{CDCl}_{3}\right): \delta 6,37(1 \mathrm{H}$, dd; $J=10,8$ e 17,4 Hz, $\mathrm{H}-4)$; 5,30-4,93 (5H, m, H-7, H-9 e H-10); 2,23-2,14 (4H, m, H-5 e H-6); 1,69 (3H, s, $\left.\mathrm{CH}_{3}-1\right) ; 1,60$ (3H, s, $\left.\mathrm{CH}_{3}-2\right) . \mathrm{RMN}{ }^{13} \mathrm{C}\left(75,5 \mathrm{MHz}, \mathrm{CDCl}_{3}\right): \delta 146,1$ (C, C8); 139,0 (CH, C-9); 132,0 (C, C-3); 124,1 (CH,C-4), 115,6 $\left(\mathrm{CH}_{2}, \mathrm{C}-7\right), 113,0(\mathrm{CH} 2, \mathrm{C}-10), 31,4\left(\mathrm{CH}_{2}, \mathrm{C}-6\right)$, 26,7 ( $\left.\mathrm{CH}_{2}, \mathrm{C}-5\right), 25,7$ (CH3, C-1); 17,7 (CH3, C-2). EM m/z (int. rel.\%): [M] 136 (2), 93 (65), 91 (15), 69 (60), 41 (100).

\section{RESULTADOS E DISCUSSÃO}

O extrato aquoso e o extrato em AcOEt, obtidos do extrato hidroalcoólico liofilizado da folhas de $P$. regnellii (Miq.) C. CD. var. pallescens (C. DC.) Yunck, foram analisados por RMN ${ }^{1} \mathrm{H}$. A análise destes extratos sugeriu que o extrato em AcOEt era constituído por uma mistura de lignanas. O fracionamento cromatográfico do extrato AcOEt (Esquema 1) resultou no isolamento de eupomatenóide-6 (1), eupomatenóide-5 (2), eupomatenóide-3 (3) e conocarpano (4). Os dados de $\mathrm{RMN}{ }^{13} \mathrm{C}$ de $\mathbf{1}$ a $\mathbf{4}$ encontram-se na Tabela 1 . A presença destas substâncias em espécies da família Piperaceae já havia sido relatada anteriormente (Chauret et. al.,1996 Freixa et. al., 2001).

As raízes de Pothomorphe umbellata (L.) Miq. da família Piperaceae, também conhecida popularmente como pariparoba, estão inscritas na primeira edição da Farmacopéia Brasileira. Elas tem sido confundidas com as de outras espécies como a P. regnelli (Miq.) C. DC. var regnellii e $P$. regnellii (Miq.) C. CD. var. pallescens (C. DC.) Yunck e são consideradas adulterantes desta espécie oficinal (Freitas, 1999). No estudo fitoquímico das folhas de $P$. regnellii (Miq.) C. CD. var. pallescens (C. DC.) Yunck, foram isoladas, pela primeira vez, as neolignanas denominadas de eupomatenóide-6 (1), eupomatenóide-5 (2), eupomatenóide-3 (3) e conocarpano (4), diferindo da composição encontrada nas raízes da Pothomorphe

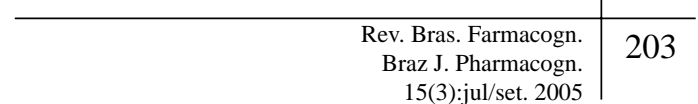


umbellata, das quais foram isolados alcalóides, flavona, diidrochalcona e esteróide (Isobe et al., 2002). As neolignanas $\mathbf{1}$ a $\mathbf{4}$ foram isoladas também das raízes de $P$. regnellii por Benevides (1999), mas a variedade dessa espécie não foi identificada. Seriam necessários estudos fitoquímicos complementares nas folhas e raízes da Pothomorphe umbellata com o objetivo de uma comparação química entre as espécies que a população utiliza como pariparoba, com o objetivo da inclusão de novas drogas na Farmacopéia Brasileira.

O óleo essencial utilizado para análise de RMN de ${ }^{1} \mathrm{H}$ e ${ }^{13} \mathrm{C}$, extraído das folhas frescas de $P$. regnellii (Miq.) apresentou um teor médio de $0,80 \% \mathrm{v} / \mathrm{p}$ com uma densidade relativa calculada em $\mathrm{d}=0,845 \mathrm{~g} / \mathrm{mL}$, sendo estes dados relatados pela primeira vez.

Através de uma análise preliminar do óleo essencial por CG/EM foi identificada um componente majoritário o $\beta$-mirceno numa concentração relativa de 70\%. A identificação desta substância foi realizada pela comparação com os espectros de massas do banco de dados do equipamento e de carbono treze com os dados da literatura, (Nemethy et. al., 1982).

Esses valores encontrados para o $\beta$-mirceno (70\%) são superiores aos encontrados por Constantin et. al. (2001), que obteve 52,6 \%, embora não tivesse citado a variedade de Piper regnellii estudada. Estudos futuros como a comparação do óleo essencial extraído de variedades de Piper regnellii deverão ser realizados. Estes resultados preliminares são um bom indicativo para estudos comparativos da variação sazonal entre estas variedades.

\section{CONCLUSÕES}

Este trabalho permitiu traçar o perfil químico de lignanas presentes nas folhas de $P$. regnellii (Miq.) C. CD. var. pallescens (C. DC.) Yunck.

A alta concentração de $\beta$-mirceno (70\%) no óleo essencial desta variedade é uma perspectiva de uma nova fonte deste metabólito secundário como matériaprima na síntese de novos medicamentos. A metodologia estabelecida para a obtenção destas neolignanas (Figura 2), a partir de folhas, poderá ser uma fonte de extração desses princípios ativos.

\section{AGRADECIMENTOS}

Os autores agradecem ao Conselho Nacional de Desenvolvimento Científico e Tecnológico, CNPq, Capacitação de Aperfeiçoamento de Pessoal de Nível Superior, Capes e ao Programa de Pós-graduação em Ciências Farmacêuticas da Universidade Estadual de Maringá pelo suporte financeiro.

\section{REFERENCIAS}

Achenbach H, Grob J, Xorge AD, Cano G, Verde JS, Brussolo
LDC, Muñoz G, Salgado F, López L 1987. Lignans, neolignanas e norneolignanas from Krameria cystisoides. Phytochemistry 26: 1159-1166.

Benevides PJC, Sartorelli P, Kato MJ 1999. Phenylpropanoids e neolignanas from Piper regnellii. Phytochemistry 52: 339-343.

Chauret DC, Bernard CB, Arnason JT, Durst T, Krishnamurty HG, Sanchezvindas P, Moreno N, Sanroman L, Poveda L 1996. Insecticidal neolignanas from Piper decurrens. J Nat Prod 59: 152-155.

Constantin MB, Sartorelli P, Limberger R, Henriques AT, Steppe M, Ferreira MJP, Ohara MT, Emerenciano VP, Kato MJ 2001. Essential oils from Piper cernuum and Piper regnellii: antimicrobial activities, analysis by GC/MS and ${ }^{13} \mathrm{C}-\mathrm{NMR}$. Planta Med 67: 771-773.

Corrêa MP 1984. Dicionário das plantas úteis do Brasil e das exóticas cultivadas. Brasília: Ministério da Agricultura, Instituto Brasileiro de Desenvolvimento Florestal, vol. V, p 687.

Cronquist A 1981. An integrated system of classification of flowering plants. New York: Columbia University Press.

Farmacopéia Brasileira 4. ed. 1988. São Paulo: Atheneu, Editora São Paulo, pt.1, vol.2.2, vol. 2.5, vol. 2.6, vol. 2.9, vol. 4.2.4, vol. 4.2.6.

Freitas PCD 1999. Atividade antioxidante de espécies medicinais da família Piperaceae: Pothomorphe umbellata (L) Miq e Piper regnellii (Miq) CDC. São Paulo, 115 p. Tese de Doutorado- Faculdade de Ciências Farmacêuticas- Universidade de Säo Paulo.

Freixa B, Vila R, Ferro EA, Adzet T, Canigueral S 2001. Antifungal principles from Piper fulvescens. Planta Med 67: 873-875.

Holetz FB, Pessini GL, Sanches NR, Cortez DAG, Nakamura CV, Dias Filho BP 2002. Screening of some plants used in the Brazilian folk medicine for the treatment of infectious diseases. Mem I Oswaldo Cruz 97: 10271031.

Isobe T, Ohsaki A, Nagata K 2002. Antibacterial constituents against Helicobacter pylori of Brazilian medicinal plant, pariparoba. Yakugaku Zasshi 122: 291-294.

Maxwell A, Dabideen D, Reynolds FW, Mclean S 1999. Neolignanas from Piper aequale. Phytochemistry 50: 499-504.

Nemethy EK, Calvin M 1982. Terpenes from Pittosporaceae. Phytochemistry 21: 2981-2982.

Pessini GL, Dias BP, Nakamura CV, Cortez DAG 2003. Antibacterial activity of extracts and neolignans from Piper regnellii (Miq.) C. DC. var. pallescens (C. DC.) Yunck. Mem I Oswaldo Cruz 98: 11151120.

Sartorelli P, Benevides PJC, Ellensohn RM, Rocha MVAF, Moreno PRH, Kato MJ 2001. Enantioseletive conversion of $p$-hydroxypropenylbenzene to $(+)$ conocarpan in Piper regnellii. Plant Sci 161: 10831088.

Snider BB, Han LN, Xie CY 1997. Synthesis of 2,3dihydrobenzofurans by $\mathrm{Mn}(\mathrm{OAc})(3)$-based oxidative cycloaddition of 2-cyclohexenones with alkenes. Synthesis of (+/-)-conocarpan. J Org Chem 62: 69786984. 